\title{
A GENERAL FORMULA FOR FUNDAMENTAL SOLUTIONS OF LINEAR PARTIAL DIFFERENTIAL EQUATIONS WITH CONSTANT COEFFICIENTS
}

\author{
GERHARD MAY
}

(Communicated by Theodore W. Gamelin)

\begin{abstract}
In this note we present a formula which furnishes particular fundamental solutions of linear partial differential equations with constant coefficients. Our construction extends an explicit formula of König (to appear) after the procedure of Malgrange (1955-1956). The crucial point is that he works with equations rather than with estimations as in the classical proof of the Malgrange-Ehrenpreis theorem. Following his ideas, we obtain fundamental solutions which are regular in the sense of Hörmander (1983); they are of basic importance. Our formula is as explicit as the zeros of a polynomial in one variable are explicit as functions of the coefficients.
\end{abstract}

The present paper will be subdivided into three parts. In the first part we present an integral formula which for suitable kernels will produce fundamental solutions for the differential operator $P(D)$ in $\mathbb{R}^{n}$,

$$
P(D):=\sum_{|\alpha| \leq m} c(\alpha) D^{\alpha}, \quad \text { where } D:=\frac{1}{i} \partial \text { and } \partial=\left(\frac{\partial}{\partial x_{1}}, \ldots, \frac{\partial}{\partial x_{n}}\right),
$$

and $P$ is a polynomial in $n$ variables with complex coefficients of precise degree $m \geq 0$. In the second part we profit from the explicit character of our formula: If its kernel satisfies certain growth conditions, then the resulting fundamental solution has additional local regularity properties. In the final part we specialize in order to obtain in particular the explicit fundamental solution of König [4] and regular fundamental solutions in the sense of Hörmander [3]. This part forms the main innovation compared with König [4].

In the sequel we use standard distributional notations as to be found, for example, in Hörmander [2]. Let $\lambda^{n}$ denote Lebesgue measure on $\mathbb{R}^{n}$, and let $\sigma$ be a finite positive Borel measure on $\mathbb{C}^{n}$ with compact support. For a bounded Borel-measurable function $M: \mathbb{R}^{n} \times \mathbb{C}^{n} \rightarrow \mathbb{C}$ we define the functional $F^{M}: \mathscr{D} \rightarrow \mathbb{C}$ on the space $\mathscr{D}$ of $\mathscr{C}^{\infty}$ functions on $\mathbb{R}^{n}$ with compact support to be

$$
\left\langle F^{M}, u\right\rangle=\frac{1}{(2 \pi)^{n}} \int_{\mathbb{R}^{n} \times \mathbb{C}^{n}} \widehat{u}(z+\zeta) M(z, \zeta) d\left(\lambda^{n} \times \sigma\right)(z, \zeta) \text { for } u \in \mathscr{D},
$$

Received by the editors January 4, 1993.

1991 Mathematics Subject Classification. Primary 35E05; Secondary 35D10. 
where $\widehat{u}$ denotes the Fourier-Laplace transform of $u$. The norm $\|\cdot \mid\|$ on $\mathscr{D}$ defined to be

$$
\||u|\|=\int_{\mathbb{R}^{n} \times \mathbb{C}^{n}}|\widehat{u}(z+\zeta)| d\left(\lambda^{n} \times \sigma\right)(z, \zeta) \quad \text { for } u \in \mathscr{D}
$$

admits an estimation $\leq C(K)\|\cdot\|_{n+1}(K)$ on the subspace $\mathscr{D}_{K} \subset \mathscr{D}$ of functions with support in the set $K$ for any compact $K \subset \mathbb{R}^{n}$, where

$$
\|u\|_{n+1}(K)=\sup \left\{\left|D^{\alpha} u(x)\right|: x \in K,|\alpha| \leq n+1\right\} \text { for } u \in \mathscr{D}_{K} .
$$

This is a consequence of the differentiability properties of the Fourier-Laplace transform; see Rudin [6, p. 196]. Therefore, $F^{M}$ is a distribution of order $\leq n+1$ on $\mathbb{R}^{n}$.

In the following theorem we state a sufficient condition in order that $F^{M}$ be a fundamental solution of $P(D)$. This condition will be verified for a special class of functions in Theorem 3 . Here and in the following we use for functions $f: \mathbb{C}^{n} \rightarrow \mathbb{C}$ the notation $\check{f}:=f(-\cdot)$.

Theorem 1. If the function $M$ satisfies the condition

$(*)$

$$
f(z)=\int_{\mathbb{C}^{n}} f(z+\zeta) \check{P}(z+\zeta) M(z, \zeta) d \sigma(\zeta)
$$

$$
\text { for all entire } f: \mathbb{C}^{n} \rightarrow \mathbb{C} \text { and } z \in \mathbb{R}^{n},
$$

then the distribution $F^{M}$ is a fundamental solution for $P(D)$.

Proof. For $u \in \mathscr{D}$ and $v:=\check{P}(D) u=P(-D) u$ we have $\widehat{v}=\check{P} \widehat{u}$, and we deduce from $(*)$ and the Fourier inversion formula

$$
\begin{aligned}
\langle\delta, u\rangle & =u(0)=\frac{1}{(2 \pi)^{n}} \int_{\mathbb{R}^{n}} \widehat{u}(z) d \lambda^{n}(z) \\
& =\frac{1}{(2 \pi)^{n}} \int_{\mathbb{R}^{n}} \int_{\mathbb{C}^{n}} \widehat{u}(z+\zeta) \check{P}(z+\zeta) M(z, \zeta) d \sigma(\zeta) d \lambda^{n}(z) \\
& =\left\langle F^{M}, P(-D) u\right\rangle=\left\langle P(D) F^{M}, u\right\rangle .
\end{aligned}
$$

This shows that $F^{M}$ is in fact a fundamental solution of $P(D)$.

In order to examine the question of local regularity of the solution $F^{M}$, we recall the relevant definitions from Hörmander [3, $\S \S 10.1$ and 10.2].

Definition 1. (i) A function $k: \mathbb{R}^{n} \rightarrow(0, \infty)$ is called a temperate weight function iff there exist constants $C>0, N \in \mathbb{N}$ such that

$$
k(z+x) \leq(1+C|z|)^{N} k(x) \text { for } z, x \in \mathbb{R}^{n} .
$$

The set of all such functions $k$ will be denoted by $\mathscr{K}$.

(ii) If $k \in \mathscr{K}$ and $1 \leq p \leq \infty$, we denote by $\mathscr{B}_{p, k}$ the space of all temperate distributions $T \in \mathscr{S}^{\prime}$ such that the Fourier transform $\hat{T}$ is a function in $\mathscr{L}_{\text {loc }}^{1}$ and

$$
\|T\|_{p, k}:=\frac{1}{(2 \pi)^{n / p}}\|k \widehat{T}\|_{\mathscr{L}^{p}}<\infty .
$$

(iii) If $k \in \mathscr{K}$ and $1 \leq p \leq \infty$, the associated local spaces $\mathscr{B}_{p, k}^{\text {loc }}$ are defined to consist of all distributions $S \in \mathscr{D}^{\prime}$ such that $u S \in \mathscr{B}_{p, k}$ for each individual $u \in \mathscr{D}$. 
An important example of a function in $\mathscr{K}$ is defined by

$$
\widetilde{Q}: \widetilde{Q}(z):=\left(\sum_{\alpha}\left|\frac{Q^{(\alpha)}(z)}{\alpha !}\right|^{2}\right)^{1 / 2} \text { for } z \in \mathbb{R}^{n}
$$

where $Q$ is a nonzero polynomial in $n$ variables with complex coefficients of precise degree $q \geq 0$ and $Q^{(\alpha)}:=\partial^{\alpha} Q$. It follows from Taylor's formula that

$$
\widetilde{Q}(z+x) \leq(1+C|z|)^{q} \widetilde{Q}(x) \text { for } z, x \in \mathbb{R}^{n}
$$

with some constant $C>0$. We note that $\tilde{Q}$ is bounded from below and that $\check{Q}^{\sim}=\widetilde{Q}^{\vee}$.

Definition 2. A fundamental solution $F$ of the differential operator $P(D)$ is called regular iff $F \in \stackrel{\mathscr{B}}{\mathscr{l}_{\infty} \mathrm{loc}} \widetilde{P}$.

Remark. The definition of the formation $\widetilde{Q}$ used here and hence Definition 2 are formally different from, but are easily checked to be equivalent to, the original definition of Hörmander [3, p. 5].

The following theorem can be viewed as a reformulation of a result of Hörmander [1, Theorem 1.3, p.30].

Theorem 2. If the function $M$ satisfies (*) and if in addition there is a constant $C>0$ such that

$$
|M(z, \zeta)| \leq \frac{C}{\check{P} \sim(z)} \text { for } z \in \mathbb{R}^{n}, \zeta \in \operatorname{supp}(\sigma),
$$

then the distribution $F^{M}$ is a regular fundamental solution for $P(D)$.

Proof. For $u \in \mathscr{D}$ and $x \in \mathbb{R}^{n}$ one has

$$
\begin{aligned}
\left|\left(u F^{M}\right)^{\wedge}(x)\right| & =\left|\left\langle u F^{M}, e^{-i\langle\cdot, x\rangle}\right\rangle\right|=\left|\left\langle F^{M}, u e^{-i\langle\cdot, x\rangle}\right\rangle\right| \\
& \leq \frac{1}{(2 \pi)^{n}} \int_{\mathbb{R}^{n} \times \mathbb{C}^{n}}\left|\left(u e^{-i\langle\cdot, x\rangle}\right)^{-}(z+\zeta)\right||M(z, \zeta)| d\left(\lambda^{n} \times \sigma\right)(z, \zeta) \\
& \leq C^{\prime} \int_{\mathbb{R}^{n} \times \mathbb{C}^{n}} \frac{|\widehat{u}(z+\zeta+x)|}{\check{P} \sim(z)} d\left(\lambda^{n} \times \sigma\right)(z, \zeta) \\
& =C^{\prime} \int_{\mathbb{R}^{n} \times \mathbb{C}^{n}} \frac{|\widehat{u}(z+\zeta)|}{\widetilde{P}(x-z)} d\left(\lambda^{n} \times \sigma\right)(z, \zeta) \\
& \leq \frac{C^{\prime}}{\widetilde{P}(x)} \int_{\mathbb{R}^{n} \times \mathbb{C}^{n}}|\widehat{u}(z+\zeta)|\left(1+C^{\prime \prime}|z|^{m}\right) d\left(\lambda^{n} \times \sigma\right)(z, \zeta) ;
\end{aligned}
$$

that is, $F^{M} \in \mathscr{B}_{\infty, \tilde{P}}^{\text {loc }}$.

Comments and consequences. (i) In terms of the spaces $\mathscr{B}_{p, k}$ it is not possible to improve the result of Theorem 2 . In fact, if $F$ is any fundamental solution of $P(D)$ with $F \in \mathscr{B}_{p, k}^{\text {loc }}$, then it follows that $\mathscr{B}_{\infty, \tilde{P}}^{\text {loc }} \subset \mathscr{B}_{p, k}^{\text {loc }}$ (Hörmander [3, remark, p. 17]).

(ii) As to the inhomogeneous equation $P(D) E=G \in \mathscr{E}^{\prime}$, the solution $E$ given by $E=F * G$, where $F$ is a regular fundamental solution, belongs to $\underset{p}{\mathscr{B} \text { loc }} \tilde{p}$ for every $k \in \mathscr{K}$ and $1 \leq p \leq \infty$ such that $G \in \mathscr{B}_{p, k}^{c}:=\mathscr{B}_{p, k} \cap \mathscr{E}^{\prime}$ (Hörmander [3, Theorem 10.3.1, p. 29]). 
(iii) If we define for polynomials $Q, R$ the relation $Q \prec R$ ( $Q$ is weaker than $R$ ) to mean that $\widetilde{Q}(z) / \widetilde{R}(z) \leq C$ for any $z \in \mathbb{R}^{n}$ with some constant $C>0$, then the above result can be rephrased as follows: If $G \in \mathscr{B}_{p, k}^{c}$ for some $k \in \mathscr{K}$ and $1 \leq p \leq \infty$ and if $Q \prec P$, then we have $Q(D) E \in \mathscr{B}_{p, k}^{\text {loc }}$ for the solution $E$ considered in (ii). Conversely, if for some $k \in \mathscr{K}$ and $1 \leq p \leq \infty$ the inhomogeneous equation has a solution $E$ such that $Q(D) E \in \mathscr{B}_{p, k}^{\text {loc }}$ for any $G \in \mathscr{B}_{p, k}^{c}$, then it follows that $Q \prec P$ (Hörmander [3, Theorem 10.3.5, p. 30]).

(iv) The special case $k=1$ and $p=2$ of (iii) yields that regular fundamental solutions are proper in the sense of Hörmander [1, p. 29]; i.e., $(Q(D) F) * \mathscr{L}_{c}^{2} \subset$ $\mathscr{L}_{\text {loc }}^{2}$ for all polynomials $Q$ with $Q \prec P$.

(v) A similar argument as in the proof of Theorem 2 shows immediately that a distribution $S$ which is continuous with respect to the norm $\|\mid \cdot\|$ is a member of $\mathscr{B}_{\infty, 1}^{\text {loc }}$. In view of Hörmander [3, Theorem 10.1.24, p. 14] these distributions are convolutors of $\mathscr{B}_{p, k}^{c}$ into $\mathscr{B}_{p, k}^{\text {loc }}$. The imporant special case $k=1$ and $p=2$ is then expressed by the relation $S * \mathscr{L}_{c}^{2} \subset \mathscr{L}_{\text {loc }}^{2}$.

In the remainder of this article we will exhibit particular integral representations $(*)$ which meet our intentions. This requires a new construction in the domain of polynomials of one and of several variables.

For a polynomial in one complex variable $Q: Q(w)=\sum_{l=0}^{q} c_{l} w^{l}$ of precise degree $q \geq 0$ we define the polynomials

$$
Q_{k}: Q_{k}(w)=c_{q} \sum_{Z} \prod_{l \notin Z}\left(w-\mu_{l}\right) \prod_{l \in Z}\left(1-\overline{\mu_{l}} w\right) \text { for } k=0, \ldots, q,
$$

where $\mu_{1}, \ldots, \mu_{q}$ are the complex zeros of $Q$ counted with multiplicities and the sum ranges over all subsets $Z$ of $\{1, \ldots, q\}$ with $k$ elements, and we set $Q_{k}:=0$ for $k>q$. One verifies that the $Q_{k}, k \geq 0$, are well defined and that

$$
\left|Q_{k}(t)\right| \leq\left(\begin{array}{l}
q \\
k
\end{array}\right)|Q(t)| \text { for } t \in \mathbb{T}:=\{w \in \mathbb{C}:|w|=1\} .
$$

By a well-known algebraic formula we have

$$
Q_{k}(0)=\frac{Q^{(k)}(0)}{k !}=c_{k} \text { for } k \geq 0 .
$$

If $Q$ is a polynomial in $n$ variables with complex coefficients of degree $\leq m$, we consider for fixed $z \in \mathbb{R}^{n}$ and $\zeta \in \mathbb{C}^{n}$

$$
\begin{aligned}
Q^{z, \zeta}: Q^{z, \zeta}(w) & =Q(z+w \zeta)=\sum_{\alpha} \frac{Q^{(\alpha)}(z)}{\alpha !}(w \zeta)^{\alpha} \\
& =\sum_{l=0}^{m}\left(\sum_{|\alpha|=l} \frac{Q^{(\alpha)}(z)}{\alpha !} \zeta^{\alpha}\right) w^{l} \text { for } w \in \mathbb{C},
\end{aligned}
$$

using the Taylor expansion of $Q$ around $z$. Then $Q^{z, \zeta}$ is a polynomial in one complex variable of precise degree denoted $-1 \leq q(z, \zeta) \leq m$, where $q(z, \zeta)=-1$ means that $Q^{z, \zeta}=0$. For $Q_{k}^{z, \zeta}:=\left(Q^{z, \zeta}\right)_{k}$ one verifies the identity

which will be used later.

$$
Q_{k}^{z, t \zeta}(w)=t^{k} Q_{k}^{z, \zeta}(t w) \quad \text { for } t \in \mathbb{T}, w \in \mathbb{C},
$$


Lemma 1. For $Q$ a polynomial in $n$ variables with complex coefficients of degree $\leq m$ and $k \geq 0$ the functions $Q_{k}: \mathbb{R}^{n} \times \mathbb{C}^{n} \rightarrow \mathbb{C}$, defined to be $Q_{k}(z, \zeta)=$ $Q_{k}^{z, \zeta}(1) / Q(z+\zeta)$ if $Q(z+\zeta) \neq 0$ and $=0$ if $Q(z+\zeta)=0$, are Borel-measurable and bounded.

Proof. (i) We introduce on the complex vector space of all polynomials of one variable the norm

$$
\|\cdot\|:\|R\|=\sum_{l=0}^{q}\left|c_{l}\right| \text { for } R: R(w)=\sum_{l=0}^{q} c_{l} w^{l} .
$$

Let $R^{0}$ be a fixed polynomial of precise degree $q \geq 0$ with distinct zeros $\nu_{1}^{0}, \ldots, \nu_{s}^{0}$, and choose $\varepsilon>0$ with $\left|\nu_{a}^{0}-\nu_{b}^{0}\right| \geq 2 \varepsilon$ for $a \neq b$. Then there exists a $\rho>0$ such that any other polynomial $R$ of degree $\leq q$ and $\left\|R^{0}-R\right\|<$ $\rho$ has exact degree $q$ and the zeros of $R$ with their respective multiplicities stay within the $\varepsilon$-neighborhoods of the zeros $\nu_{j}^{0}$. This follows from Rouche's theorem via the condition $\left|R^{0}(w)-R(w)\right|<\left|R^{0}(w)\right|$ on the $\varepsilon$-circles around the individual $\nu_{j}^{0}(j=1, \ldots, s)$. Therefore, for the polynomials $R$ of some fixed degree the polynomials $R_{k}, k \geq 0$, are continuous functions of both the argument and the coefficients of $R$.

(ii) For fixed $z^{0} \in \mathbb{R}^{n}, \zeta^{0} \in \mathbb{C}^{n}$, and $\rho>0$ one can find $\gamma>0$ such that $\left\|Q^{z^{0}, \zeta^{0}}-Q^{z, \zeta}\right\|<\rho$ for $z \in \mathbb{R}^{n}, \zeta \in \mathbb{C}^{n}$, with $\left|\left(z^{0}, \zeta^{0}\right)-(z, \zeta)\right|<\gamma$.

(iii) We define for $q=0,1, \ldots, m$ the sets

$$
U(q):=\left\{(z, \zeta) \in \mathbb{R}^{n} \times \mathbb{C}^{n}: Q(z+\zeta) \neq 0 \text { and } q(z, \zeta) \geq q\right\},
$$

and we set $U(-1):=\mathbb{R}^{n} \times \mathbb{C}^{n}, U(m+1):=\varnothing$. These sets are all open, and they decrease as $q$ increases. We conclude from (i) and (ii) that on $U(q) \backslash U(q+1)$ the functions $Q_{k}^{\cdot} \cdot{ }^{\circ}(\cdot)$ are continuous, which implies the measurability of $Q_{k}$. In view of the estimation

$$
\left|Q_{k}^{z, \zeta}(t)\right| \leq\left(\begin{array}{c}
q(z, \zeta) \\
k
\end{array}\right)\left|Q^{z, \zeta}(t)\right| \text { for } z \in \mathbb{R}^{n}, \zeta \in \mathbb{C}^{n}, t \in \mathbb{T},
$$

the functions $Q_{k}$ are bounded by $\left(\begin{array}{c}m \\ k\end{array}\right)$ on $\mathbb{R}^{n} \times \mathbb{C}^{n}$.

For $r=0, \ldots, m$ we now define $M_{r}: \mathbb{R}^{n} \times \mathbb{C}^{n} \rightarrow \mathbb{C}$ to be

$$
M_{r}(z, \zeta)=\frac{\sum_{k=r}^{m} \check{P}_{k}(z, \zeta) \overline{\sum_{|\alpha|=k} \frac{\check{P}^{(\alpha)}(z)}{\alpha !} \zeta^{\alpha}}}{\sum_{|\alpha|=r}^{m}\left|\frac{\check{P}_{(\alpha)}(z)}{\alpha !}\right|^{2}} \text { for } z \in \mathbb{R}^{n}, \zeta \in \mathbb{C}^{n} .
$$

Lemma 1 and the Cauchy-Schwarz inequality show that the $M_{r}$ are bounded Borel-measurable functions and that

$$
\left|M_{r}(z, \zeta)\right| \leq C\left(\sum_{|\alpha|=r}^{m}\left|\frac{\check{P}^{(\alpha)}(z)}{\alpha !}\right|^{2}\right)^{-1 / 2} \quad \text { for } z \in \mathbb{R}^{n}, \zeta \in \mathbb{T}^{n},
$$

with some constant $C>0$. Furthermore, we take the measure $\tau^{n}:=\tau \times \cdots \times \tau$, where $\tau$ is normalized arc length on $\mathbb{T}$. In order to verify that the associated distributions $F^{M_{r}}$ are fundamental solutions for $P(D)$, we have to check that $(*)$ is fulfilled. We first isolate the decisive one-variable integral representation as a lemma. 
Lemma 2. For $g: \mathbb{C} \rightarrow \mathbb{C}$ an entire function and $Q$ a polynomial of one complex variable one has

$$
g(0) \frac{Q^{(k)}(0)}{k !}=\int_{\mathbb{T}} g(t) Q_{k}(t) d \tau(t) \quad \text { for } k \geq 0 .
$$

Proof. We apply the Cauchy formula to the function $G: G(w)=g(w) Q_{k}(w)$ for $w \in \mathbb{C}$ and note that $Q_{k}(0)=Q^{(k)}(0) / k !$.

Theorem 3. For $f: \mathbb{C}^{n} \rightarrow \mathbb{C}$ an entire function and $P$ a polynomial in $n$ variables of precise degree $m \geq 0$ one has

$$
f(z)=\int_{\mathbb{T}^{n}} f(z+\zeta) \check{P}(z+\zeta) M_{r}(z, \zeta) d \tau^{n}(\zeta) \text { for } z \in \mathbb{R}^{n} \text { and } r=0, \ldots, m \text {. }
$$

Proof. We apply Lemma 2 for fixed $z \in \mathbb{R}^{n}, \zeta \in \mathbb{C}^{n}$ to the functions $g^{z, \zeta}$ : $\mathbb{C} \rightarrow \mathbb{C}, g^{z, \zeta}(w)=f(z+w \zeta)$, and $Q^{z, \zeta}$, where $Q=\check{P}$ for $k \geq 0$. This gives

$$
f(z) \sum_{|\alpha|=k} \frac{Q^{(\alpha)}(z)}{\alpha !} \zeta^{\alpha}=\int_{\mathrm{T}} f(z+t \zeta) Q_{k}^{z, \zeta}(t) d \tau(t) .
$$

If we multiply this by $\overline{\sum_{|\alpha|=k} \frac{Q^{(\alpha)}(z)}{\alpha !} \zeta^{\alpha}}$, we obtain

$$
f(z)\left|\sum_{|\alpha|=k} \frac{Q^{(\alpha)}(z)}{\alpha !} \zeta^{\alpha}\right|^{2}=\int_{\mathbb{T}} f(z+t \zeta) Q_{k}^{z, \zeta}(t) \overline{\sum_{|\alpha|=k} \frac{Q^{(\alpha)}(z)}{\alpha !} \zeta^{\alpha}} d \tau(t) .
$$

Integration over $\mathbb{T}^{n}$ with respect to $\tau^{n}$ and the use of Cauchy's formula lead to

$$
f(z) \sum_{|\alpha|=k}\left|\frac{Q^{(\alpha)}(z)}{\alpha !}\right|^{2}=\int_{\mathbb{T}^{n}} \int_{\mathbb{T}} f(z+t \zeta) Q_{k}^{z, \zeta}(t) \overline{\sum_{|\alpha|=k} \frac{Q^{(\alpha)}(z)}{\alpha !} \zeta^{\alpha}} d \tau(t) d \tau^{n}(\zeta) .
$$

From the Fubini theorem and the identity $Q_{k}^{z, \zeta}(t w)=Q_{k}^{z, t \zeta}(w) \bar{t}^{k}$ for $t \in \mathbb{T}$, $w \in \mathbb{C}$ we conclude

$$
\begin{aligned}
f(z) \sum_{|\alpha|=k}\left|\frac{Q^{(\alpha)}(z)}{\alpha !}\right|^{2} & =\int_{\mathbb{T}} \int_{\mathbb{T}^{n}} f(z+t \zeta) Q_{k}^{z, t \zeta}(1) \overline{\sum_{|\alpha|=k} \frac{Q^{(\alpha)}(z)}{\alpha !}(t \zeta)^{\alpha}} d \tau^{n}(\zeta) d \tau(t) \\
& =\int_{\mathbb{T}^{n}} f(z+\zeta) Q_{k}^{z, \zeta}(1) \overline{\sum_{|\alpha|=k} \frac{Q^{(\alpha)}(z)}{\alpha !} \zeta^{\alpha}} d \tau^{n}(\zeta)
\end{aligned}
$$

where in the latter equation we used the fact that the inner integral on the left side is independent of $t \in \mathbb{T}$. The assertion follows after summation over $k=r, \ldots, m$.

The case $r=0$ gives a regular fundamental solution, whereas for $r=m$ we obtain the explicit formula constructed by König [4], which reads

$$
\begin{aligned}
& \left\langle F^{M} m, u\right\rangle \\
& \quad=\frac{1}{(2 \pi)^{n}} \int_{\mathbf{R}^{n} \times \mathbb{T}^{n}} \widehat{u}(z+\zeta) \frac{\llbracket \check{P}(z+\zeta) \rrbracket(-1)^{m} \sum_{|\alpha|=m} c(\alpha) \zeta^{\alpha}}{\sum_{|\alpha|=m}|c(\alpha)|^{2}} d\left(\lambda^{n} \times \tau^{n}\right)(z, \zeta)
\end{aligned}
$$

for $u \in \mathscr{D}$, 
where $\llbracket \cdot \rrbracket: \mathbb{C} \rightarrow \mathbb{C}$ is defined to be $\llbracket w \rrbracket=\bar{w} / w$ for $w \neq 0$ and $=0$ for $w=0$. Here we did not use the multinomial formula as König [4] did in order to represent his fundamental solution as a sum of derivatives of continuous functions.

\section{ACKNOWLEDGEMENT}

The author thanks Professor Heinz König for his advice during the course of this work and the referee for valuable remarks and additional hints to the literature.

\section{REFERENCES}

1. L. Hörmander, Local and global properties of fundamental solutions, Math. Scand. 5 (1957), 27-39.

2. __ The analysis of linear partial differential operators. I, Springer-Verlag, New York, 1983.

3. __ The analysis of linear partial differential operators. II, Springer-Verlag, New York, 1983.

4. H. König, An explicit formula for fundamental solutions of linear partial differential equations with constant coefficients, Proc. Amer. Math. Soc. (to appear).

5. B. Malgrange, Existence et approximation des solutions des équations aux dérivées partielles et des équations de convolution, Ann. Inst. Fourier (Grenoble) 6 (1955-1956), 271-355.

6. W. Rudin, Functional analysis, McGraw-Hill, New York, 1973.

Universität des SaArlandes, Fachbereich Mathematik, D-66041 SaArbrücken, GerMANY 